\title{
La modélisation de l'œil humain : de nouvelles perspectives pour la simulation industrielle
}

Simuler de manière précise et fiable ce que verrait une personne immergée dans une scène 3D est un enjeu capital pour le monde industriel. Cependant, les problématiques de secteurs tels que l'automobile ou I'aéronautique ne pourront être résolues qu'à une seule condition : arriver à une image simulée très proche de celle que la personne obtiendrait dans la réalité, en tenant compte de facteurs humains comme l'âge et les déficiences visuelles pour que les ingénieurs puissent concevoir des produits en se mettant à la place de tous types d'utilisateur : comment l'éclairage et les écrans d'un tableau de bord de voiture seront-ils perçus par une personne de 70 ans, de jour, de nuit ou avec le soleil de face ? C'est pour répondre à ces questions qu'un modèle d'œil humain couplé à des simulations physiques de propagation de la lumière est développé depuis plusieurs années.

$$
\begin{aligned}
& \text { Laurent FOURNIER } \\
& \text { Optis } \\
& \text { Ifournier@optis-world.com }
\end{aligned}
$$

a simulation de la propagation de Lla lumière intégrant les propriétés physiques de l'interaction lumièrematière permet d'évaluer la quantité de lumière reçue par un capteur placé dans une scène 3D virtuelle. La connaissance de la composition spectrale du signal reçu permet alors de simuler la réponse de plusieurs types de capteurs : photodiodes, capteurs CCD, photomultiplicateurs...

La simulation de ce qui est sans doute le plus performant des capteurs optiques, l'œil humain, vise à afficher sur un écran ce que verrait une personne immergée dans la scène 3D pour, finalement, pouvoir se mettre à sa place en tenant compte de son âge et éventuellement de ses déficiences visuelles.

\section{L'œil humain}

\section{Propriétés optiques}

Pour modéliser l'œil humain, il faut tout d'abord prendre en compte les propriétés du système optique constitué par la cornée, I'iris, le cristallin, I'humeur aqueuse et le corps vitré.

La taille de la pupille s'adapte en permanence aux conditions lumineuses afin de limiter la quantité de lumière reçue par la rétine. À la traversée de la cornée, du cristallin et des couches supérieures de la rétine, une partie de la lumière est diffusée et génère un voile laiteux que l'on peut facilement observer en présence de sources lumineuses de forte intensité. Le halo coloré observé autour de certaines sources lumineuses s'explique par la présence de fibres radiales à la périphérie du cristallin qui se comportent comme un réseau optique. Le halo n'est visible que pour des conditions d'observation avec de très faibles niveaux de luminance, c'est-à-dire lorsque la taille de la pupille est maximale.

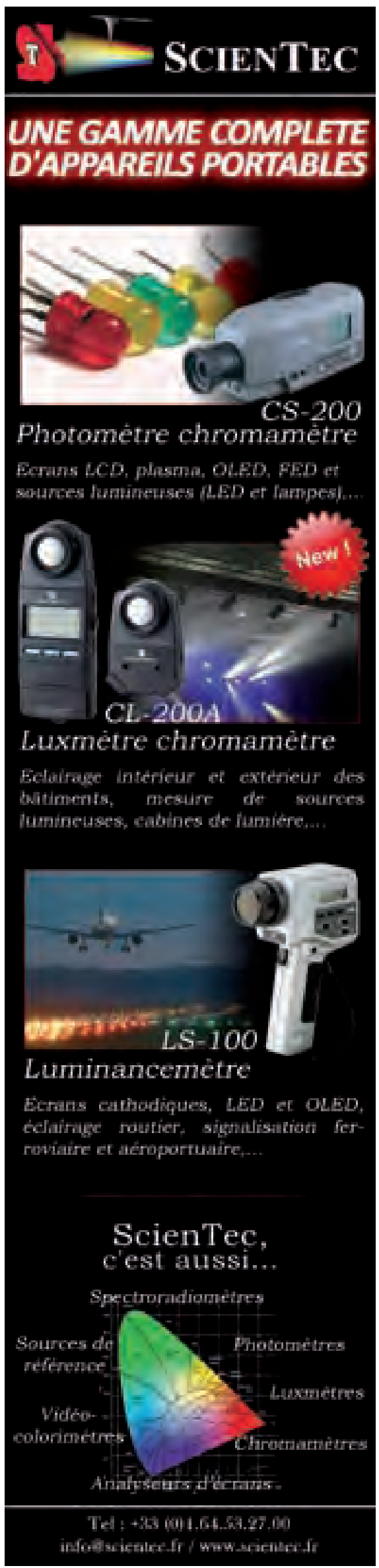

N48 - Septembre/octobre 2010 - Thotoniques 
On peut également observer, autour des sources de faibles étendues angulaires, des traits lumineux radiaux, dus à une diffusion cohérente de la lumière par des particules présentes dans les milieux oculaires [1].

\section{Réponse de la rétine}

Il faut également tenir compte de la réponse de la rétine et plus précisément des photorécepteurs (cônes et bâtonnets) qui la composent, en fonction notamment de la luminance.

Les cônes sont essentiellement situés au centre de la rétine, dans une zone appelée fovéa. La très forte densité de cônes dans la fovéa nous confère une très bonne acuité visuelle en vision centrale. L'existence de trois types de cônes à sensibilités spectrales distinctes, centrées sur 450, 540 et $610 \mathrm{~nm}$, nous permet de pouvoir distinguer les couleurs. Les bâtonnets, qui ont une sensibilité beaucoup plus élevée que celle des cônes, sont répartis sur toute la rétine, hors fovéa, mais avec une densité beaucoup moins importante. Ils ne permettent pas de distinguer les couleurs, mais sont plus sensibles à la lumière diffusée par les milieux oculaires.

En dessous de 0,005 candela par mètre carré $\left(\mathrm{Cd} / \mathrm{m}^{2}\right)$, la réponse des bâtonnets est prépondérante par rapport à celle des cônes : on parle de vision scotopique. L'acuité visuelle pour de très faibles luminances est donc meilleure en périphérie qu'au centre de la rétine, mais le voile laiteux présent autour des sources lumineuses est de ce fait plus intense la nuit que le jour.

Dans la vision photopique, au-dessus de $6 \mathrm{Cd} / \mathrm{m}^{2}$, les bâtonnets sont totalement saturés et ne contribuent plus au signal. Entre ces deux valeurs de luminance, on parle de vision mésopique.

Le temps d'adaptation des bâtonnets aux conditions lumineuses est beaucoup plus long que celui des cônes. Ainsi, lors du passage d'une zone très lumineuse à une zone très sombre, le temps de désaturation des bâtonnets peut atteindre trente minutes. L'adaptation d'une zone très sombre à une zone très lumineuse en revanche dépasse rarement quelques secondes.

\section{Âge de l'observateur}

Les caractéristiques de la vision humaine que nous venons d'évoquer dépendent bien évidemment de l'âge de l'observateur car, au fur et à mesure que l'observateur vieillit, les temps d'adaptation s'allongent, les éblouissements se font de plus en plus intenses et la vision des contrastes se dégrade.

\section{Modélisation}

Le modèle d'œil humain que nous avons développé pour simuler les caractéristiques de la vision humaine décrites cidessus est intégré à un logiciel de simu- lation de la propagation de lumière baptisé SPEOS, lui-même intégré CAO* pour permettre aux industriels d'optimiser l'ergonomie visuelle de leurs interfaces homme-machine à partir de leurs modèles existants.

Ce logiciel permet de simuler la propagation des rayons lumineux dans l'œil (figure 1) pour obtenir des simulations de la vision réelle d'un observateur ayant ses propres caractéristiques oculaires.

\section{La simulation photométrique}

La première étape dans la simulation doit décrire précisément le niveau de lumière dans la scène. Ceci nécessite une approche photométrique, fondamentale pour générer le signal nécessaire pour obtenir la réponse de l'œil. L'outil permet de maîtriser, et de simuler avec précision, l'émission des sources, y compris la lumière ambiante, la propagation de la lumière, l'interaction avec les surfaces et matériaux avec une gestion spectrale des données.

Les propriétés optiques des surfaces et des matériaux sont appliquées au modèle CAO à partir d'une bibliothèque de données des fabricants qui se présente sous la forme de fichiers de BRDF (Bidirectional Reflectance Distribution Function) (figure 2). Elles peuvent également être définies par l'utilisateur ou mesurées à l'aide d'un photogoniomètre.

\section{${ }^{*}$ SPEOS est intégré dans les logiciels de CAO CATIA V5 ${ }^{\circledR}$, SolidWorks ${ }^{\circledR}$ et Pro/ENGINEER ${ }^{\circledR}$.}

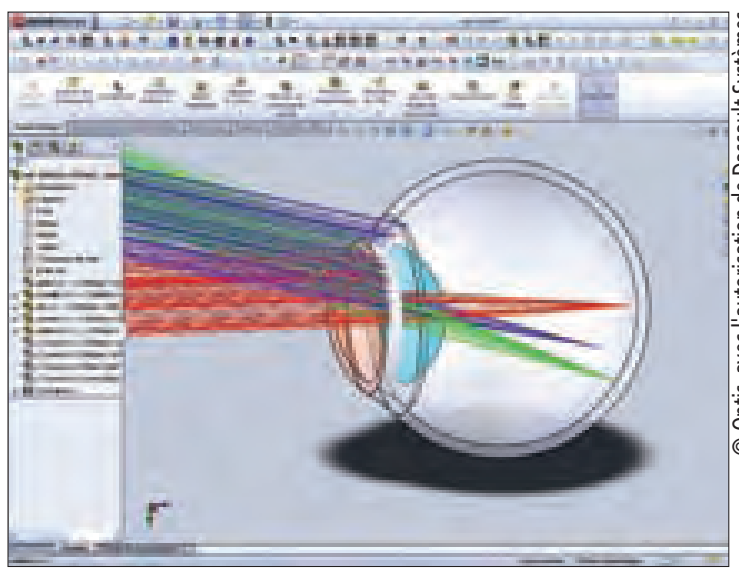

Figure 1. Simulation de la propagation de rayons lumineux à travers les milieux oculaires.

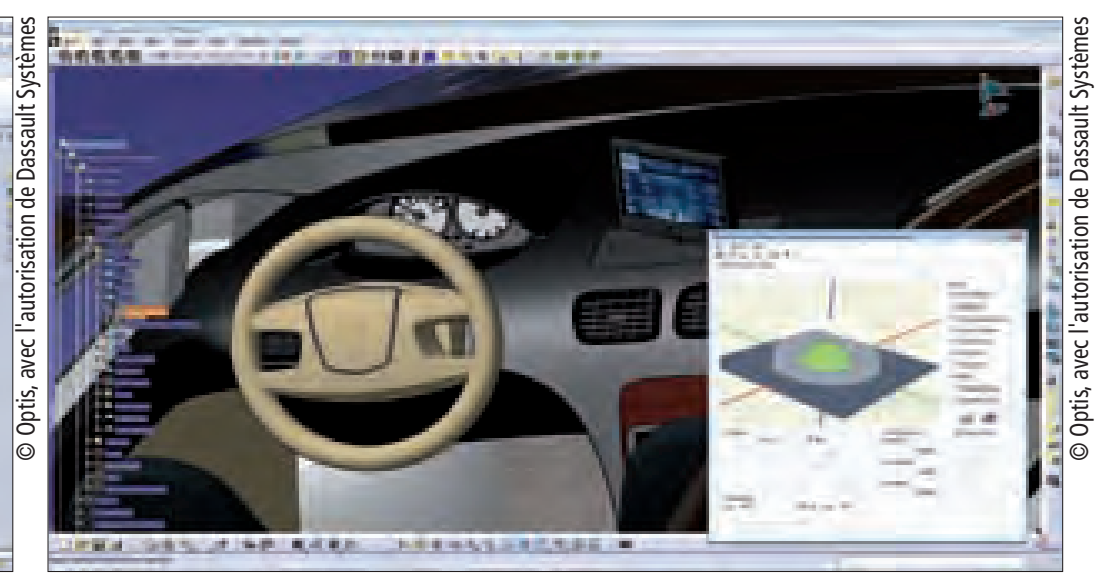

Figure 2. Sélection du matériau du volant (simulation Optis, modèle Dassault Systèmes). 
Les sources lumineuses telles que les phares ou les écrans LCD sont ensuite définies à partir d'une géométrie et d'une distribution d'intensité spectrale $(\mathrm{Cd} / \mathrm{nm})$. La dernière étape de préparation consiste à ajouter la vision humaine en configurant un capteur qui représente les yeux d'un observateur standard ou ayant des caractéristiques spécifiques.

Ensuite, il s'agit de lancer les simulations pour obtenir les résultats quantitatifs photométriques et colorimétriques pour n'importe quel point de la scène, et également qualitatifs : les rendus réalistes et scientifiquement corrects du point de vue de l'observateur.

\section{Affichage des résultats}

L'affichage sur un écran des résultats simulés représente un problème non négligeable dans la mise au point d'un modèle d'œil.

L'œil humain est en effet capable de distinguer des luminances allant de $10^{-6}$ à plus de $10^{5} \mathrm{Cd} / \mathrm{m}^{2}$, mais les écrans sur lesquels nous pouvons afficher les résultats ne disposent pas d'une telle dynamique : ils permettent d'afficher des valeurs de luminance allant d'environ un dixième à quelques centaines de candelas par mètre carré.

Les résultats doivent donc être compressés tout en conservant un contraste entre deux zones affichées sur l'écran identique à celui qu'obtiendrait l'observateur immergé dans la scène.

Ainsi, un objet qui ne serait pas visible dans la scène réelle ne doit pas l'être lorsque le résultat de la simulation est affiché sur l'écran.

Pour ce faire, nous construisons un histogramme de luminance du résultat obtenu sur la rétine. L'image sur la rétine est échantillonnée en zone ayant un angle d'observation d'environ un degré, ce qui correspond à la zone couverte par la fovéa. La luminance moyenne de ces zones est ensuite calculée pour construire l'histogramme. Ceci nous permet d'accorder le maximum de dynamique de l'écran aux zones de luminance de l'image les plus étendues spatialement. Nous nous assurons dans le même temps que le contraste affiché est bien en corrélation avec celui qui serait perçu dans la scène réelle.

\section{Exemple de résultats}

À partir d'une scène initiale, la modélisation permet de calculer la luminance perçue par la rétine, la décomposition spectrale de la lumière et les coordonnées colorimétriques de chaque point de l'image dans des conditions particulières.

Les images de la figure 3 montrent la simulation des effets de l'intensification de l'éblouissement et de l'affaiblissement de la vision des contrastes avec l'âge du conducteur. On constate que le conducteur de 70 ans a une moins bonne visibilité des bas-côtés de la route, notamment parce que son éblouissement est beaucoup plus intense que celui observé par le conducteur de 20 ans. Le conducteur le plus âgé $\gg$

\section{Modulateurs New Focus ${ }^{\mathrm{T}}$} Plus de choix. Plus d'efficacité. Plus de fréquence.

\section{Demandez plus avec la famille de modulateurs New Focus}

Avec les modulateurs New Focus, MICRO-CONTROLE Spectra-Physics vous propose le choix dont vous avez besoin et vous apporte les performances et la qualité exceptionnelles que vous attendez. Nous proposons aussi divers contrôleurs, systèmes de hachage et accessoires pour vous aider à réaliser votre montage rapidement et avec efficacité.

\section{Caractéristiques des produits}

- Large gamme de modulateurs électro-optiques de phase et d'amplitude, large bande ou sur une fréquence de résonance particulière

- Cristaux de KTP ou de niobate de lithium de la meilleure qualité

- Gamme de longueur d'onde de 500 nm à 1600 nm, pour des fréquences atteignant 9,2 GHz

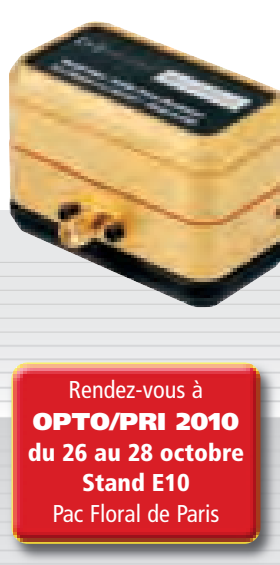

Pour en savoir plus, visitez www.newport.com/newfocus Veuillez nous appeler pour connaître les prix et les disponibilités.

MICRO-CONTROLE Spectra-Physics S.A.S

1, rue Jules Guesde - Bâtiment B Tél.: 01.60.91.68.68

Zl. du Bois de l'Épine - BP189 Fax: 01.60.91.68.69

91006 Évry CEDEX

e-mail: france@newport.com

www.newfocus.com

【 $\nu 】$ New Focus

C 2010 Newport Corporation. 

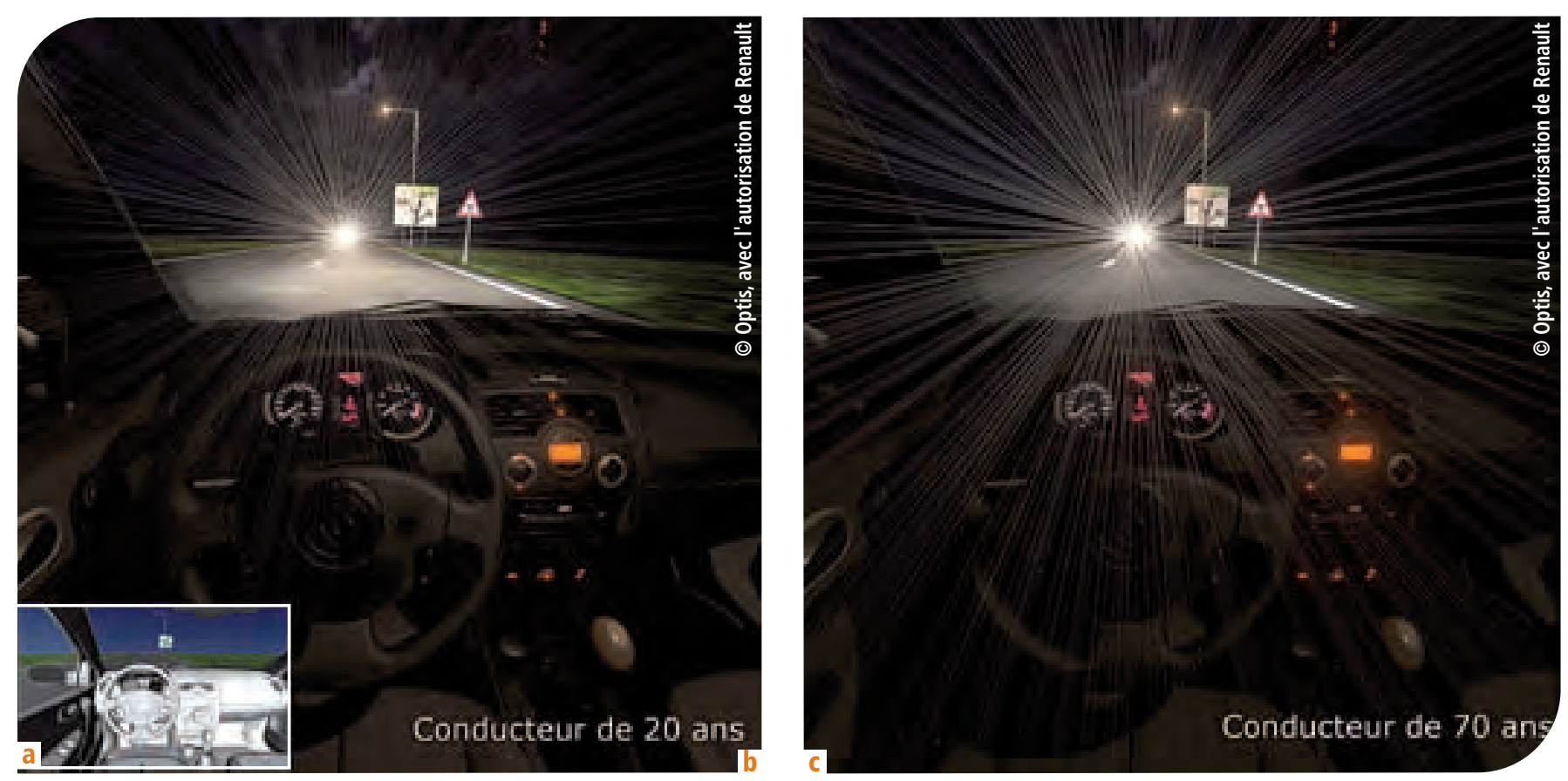

Figure 3. Simulation de l'évolution de l'éblouissement en fonction de l'âge : image de base sur laquelle la CA0 a été réalisée (a), scène vue par un conducteur de 20 ans (b) et par un conducteur de 70 ans (c).

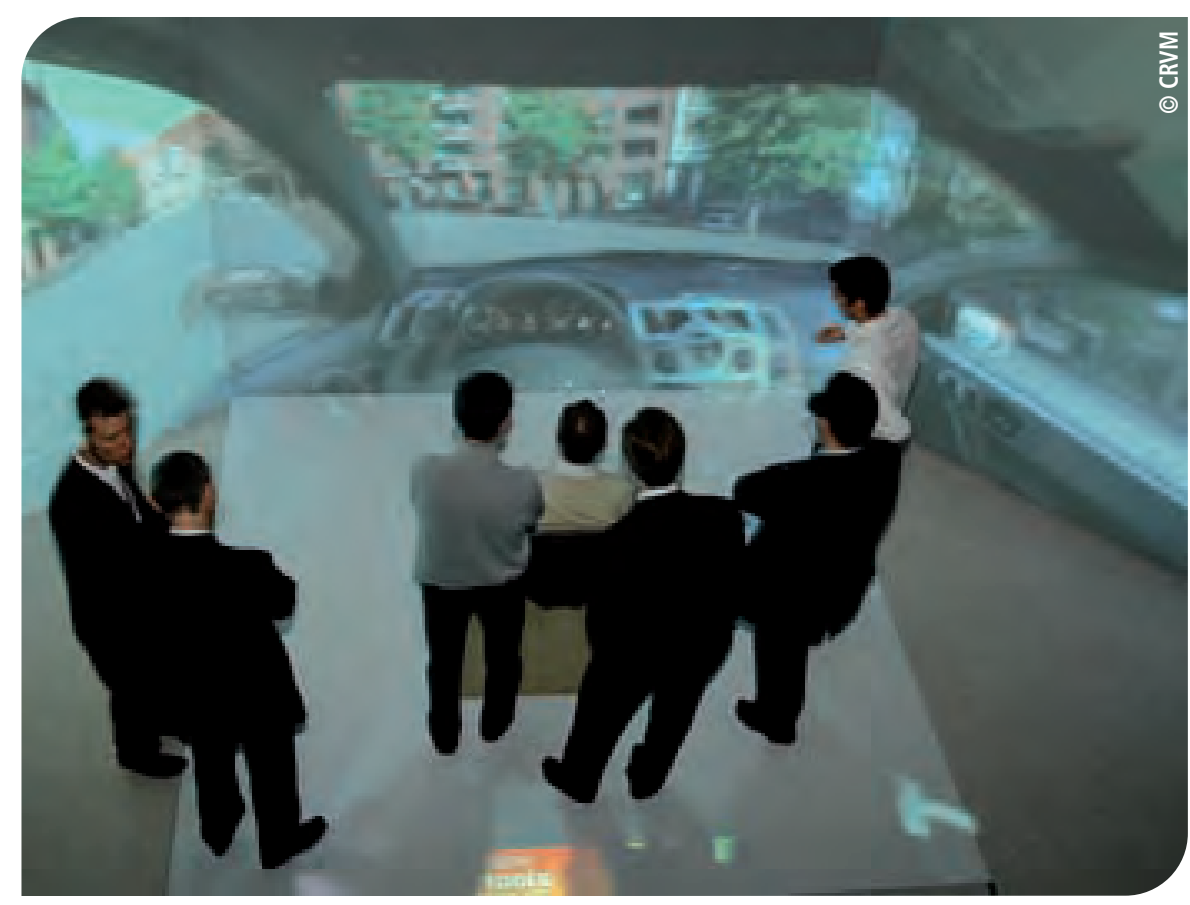

Figure 4. Affichage de résultats de simulation de SPEOS en vision stéréoscopique au Centre de réalité virtuelle de la Méditerranée.

est également plus ébloui par les éclairages du tableau de bord.

\section{Applications}

Les simulations de la vision réelle permettent aux ingénieurs et concepteurs de se mettre à la place de n'importe quel utilisateur pour trouver la solution opti- dans le pare-brise ou d'un voyant lumineux qui serait peu lisible pour cette catégorie d'utilisateur. Plus ces anomalies sont corrigées tôt dans le processus de développement, plus les économies de temps et d'argent seront importantes.

\section{Perspectives}

Les acteurs du monde industriel ont de plus en plus recours à la réalité virtuelle dans les phases de conception de leurs produits (figure 4) car elle permet d'identifier instantanément les erreurs de conception, d'ergonomie et de sécurité.

Les enjeux futurs sont donc d'intégrer la modélisation de l'œil humain dans les systèmes immersifs temps réel pour simuler la vision stéréoscopique tout en permettant à l'utilisateur d'interagir avec l'environnement virtuel dans lequel il sera immergé.

male pour satisfaire tous les utilisateurs, ou une solution adaptée à une cible particulière.

Ils vont par exemple pouvoir concevoir les affichages d'un tableau de bord de voiture en se mettant à la place d'un conducteur âgé et ainsi se rendre compte d'une réflexion du tableau de bord

\section{Référence}

1. Thomas JTP van den Berg, Michiel PJ Hagenouw, Joris E Coppens. The ciliary corona: physical model and simulation of the fine needles radiating from point light sources. Investigative Ophthalmology \& Visual Science, July 2005, Vol. 46, No. 7. 